\title{
ESTRATEGIAS DISCURSIVAS Y OPINIÓN DEL ALUMNADO SOBRE LA INTERACCIÓN CON EL DOCENTE EN FORMACIÓN MÉDICA
}

\author{
José Luis Cruz González y Edgardo Ruíz Carrillo \\ FES Iztacala UNAM \\ México
}

\begin{abstract}
RESUMEN
Con el objetivo de identificar la relación entre estrategias discursivas hechas entre docentes y alumnos, y la percepción del alumnado respecto a la interacción, las secuencias de discurso fueron identificadas y comparadas con las valoraciones del alumno mediante el Inventario de Interacción Profesor-Alumno (IIP-A). Los participantes fueron 144 alumnos correspondientes a cinco grupos de la carrera de Medicina de una institución pública de educación superior (IPES) y cinco profesores de la misma asignatura teórica. Se encontró que los docentes permitían a los alumnos ser expositores durante las clases, pero no mejoraba la impresión del alumnado respecto a la interacción; era la cohesión dentro del discurso, al mantener una comunicación constante entre ambos roles, donde la opinión mejoraba. Se propone fomentar la retroalimentación efectiva y la evaluación formativa docente para mejorar e incrementar las herramientas discursivas que permitan una comunicación abierta y enriquecedora con el alumnado.
\end{abstract}

Palabras Clave:

estrategias discursivas, interacción profesor-alumno, formación médica, análisis del discurso, evaluación formativa.

\section{DISCURSIVE STRATEGIES AND OPINION OF THE STUDENTS ABOUT THE INTERACTION WITH THE TEACHER IN MEDICAL TRAINING}

\begin{abstract}
With the objective of identify the relationship between discursive strategies carried out between teachers and students, and the student's perception regarding this interaction, the discursive sequences were identified, and compared with the students' evaluations, using the Inventario de Interacción Profesor-Alumno (IIP-A). The participants were 144 students from five groups in the medical career of a Institución Pública de Educación Superior (IPES), and five professors from the same theoretical subject. It was found that the teachers allowed the students to be speakers during the classes, but it did not necessarily improve the students' impression of the interaction, it was the cohesion within the discourse, by maintaining constant communication between both roles, where the opinion improved. It is proposed to promote effective feedback and teacher training evaluation, to improve and increase the discursive tools that allow open and enriching communication with students.
\end{abstract}

Keywords:

discursive strategies, teacher-student interaction, medicine teaching, discourse analysis, formative evaluation.

Bitácora del Artículo:

| Recibido: 8 de Mayo de 2020 | Aceptado: 07 enero de 2021 | Publicado en línea: Enero - Junio de 2021 | 
Artículo Teórico | Estrategias discursivas y opinión del alumnado... Cruz-González eZ Ruíz Carrillo

Autoría y Derechos de Propiedad Intelectual

\title{
ESTRATEGIAS DISCURSIVAS Y OPINIÓN DEL ALUMNADO SOBRE LA INTERACCIÓN CON EL DOCENTE EN FORMACIÓN MÉDICA
}

\author{
José Luis Cruz González y Edgardo Ruíz Carrillo \\ FES Iztacala UNAM \\ México
}

\author{
José Luis Cruz González \\ FES- Iztacala, UNAM \\ Correo: jlcgsiruscapella@gmail.com
}

Licenciatura en Psicología, alumno de doctorado en Psicología de la Educación y del Desarrollo por la UNAM. Becario de investigación/ colaborador en los proyectos de PAPIME PE304715 Y PE300617 enfocado al análisis del discurso en el ámbito educativo.

\author{
Edgardo Ruíz Carrillo \\ FES- Iztacala, UNAM \\ Correo:edgardo@unam.mx
}

Doctor en Investigación Psicológica, Universidad Iberoamericana. Distinción como Investigador Nacional Nivel 1 (Sistema Nacional de Investigadores Conacyt). Ratificación para seguir siendo del Padrón de Tutores del Plan de Estudio de Doctorado en Psicología de la UNAM.

\section{CONTRIBUCIÓN DE LOS AUTORES}

José Luis Cruz González realizó la documentación científica, grabación y recopilación de datos, aplicación de instrumentos, análisis de resultados, y elaboración de discusión y conclusiones. | Edgardo Ruíz Carrillo estuvo a cargo de la documentación científica, análisis de resultados y elaboración de la discusión

\section{AGRADECIMIENTOS}

La investigación fue apoyada por el Consejo Nacional de Ciencia y Tecnología (Conacyt) por medio de una beca nacional otorgada al primer autor con número de CVU 771380 y número de registro de beca 609130.

\section{DATOS DE FILIACIÓN DE LOS AUtORES}

Facultad de Estudios Superiores Iztacala, UNAM

Copyright: @ 2021 Cruz-Gonázalez, J.L.; \& Ruíz-Carrillo, E.

Este es un artículo de acceso abierto distribuido bajo los términos de la licencia Creative Commons Reconocimiento-NoComercial 4.0 Internacional, por lo que su contenido gráfico y escrito se puede compartir, copiar y redistribuir total o parcialmente sin necesidad de permiso expreso de sus autoras con la única condición de que no se puede usar con fines directamente comerciales y los términos legales de cualquier trabajo derivado deben ser los mismos que se expresan en la presente declaración. La única condición es que se cite la fuente con referencia a la Revista Digital Internacional de Psicología y Ciencia Social y a sus autoras. 


\section{TABLA DE CONTENIDO}

MÉTODO

Participantes, 28

Materiales, 28

Consideraciones éticas, 29

Procedimiento

Mediciones, 29

\section{ANÁLISIS ESTADÍSTICOS}

RESULTADOS

Estadística descriptiva del IIP-A en la muestra completa, 32

Frecuencia de eventos registrados de la muestra completa, 32

Correlación de categorías de discurso, 32

Discusión

CONCLUSIONES

REFERENCIAS 
$\mathrm{E}$ sta investigación se desarrolló para encontrar la relación entre las estrategias discursivas usadas por el docente y la opinión de los alumnos respecto a las clases tomadas con él. A continuación se hará una revisión de la literatura disponible para entender la evolución teórica de la interacción en el aula, los interlocutores involucrados y las comprensiones respecto a la relevancia del discurso en el salón de clases.

Para iniciar se debe entender que alumno y docente ocupan formas únicas de acción dentro del salón de clases. Coll y Miras (1993) citan a Hargreaves (1977), quien definió dos sub-roles básicos que todo profesor desempeña en el aula: el de instructor y el de mantenedor de la disciplina. Para Hargreaves (1977, citado en Coll y Miras, 1993) el sub-rol de instructor se relaciona con los esfuerzos por conseguir que los alumnos aprendan y se asocia con las expectativas de comportamiento vinculadas al establecimiento y desarrollo del currículum, mientras que ser encargado de la disciplina se relaciona con la gestión de actividades, así como el control de normas y reglas de conducta. Al ser ineludibles el docente no puede abandonar alguno de ellos, sin embargo puede prestarles importancia de manera diferente. Nassaji y Wells (2000) explican de modo similar que el docente tiene un rol doble como conocedor primario y manager o facilitador en el salón, donde mediante mecanismos o estrategias discursivas el docente confirma el conocimiento del estudiante como correcto e incorrecto (evaluaciones), haciendo reparaciones si es necesario (retroalimentando), asignando derechos de habla (preguntando a personas en específico o reanudando el tema) y revisando el entendimiento de los estudiantes (preguntas de sondeo o monitoreo).

Estas características toman forma en la teoría de la actividad de Engestrom (1993, en Lin, 2007), el cual define un sistema de actividad, como la enseñanza escolarizada, a manera de un conjunto de formaciones socio-histórico-culturales, constituidas por personas e instituciones que evolucionan a través del tiempo, autorreproductoras hasta que contradicciones internas (multivocidad o pluralidad de voces) generan fuerza de cambio y transformación, y se encuentran mediadas por herramientas culturales simbólicas y físicas cuyos usos son socialmente compartidos y de naturaleza colectiva.

Esto se vincula a lo expresado por Pozo (2014), quien en su estudio sobre la práctica docente encontró una tendencia de ayudar para aprender de manera similar a como el experto (docente) aprendió, haciendo relevante el analizar las concepciones de la docencia y herramientas que ha adquirido el docente para desempeñar esta práctica porque las preferencias evaluativas y enfoques de aprendizaje son correlativos a la manera en que el docente enseña, como ya fue confirmado por Argos, Ezquerra, Osorio, Salvador y Castro (2013).

Gutiérrez-Giraldo y López-Isaza (2011) indagaron esta interactividad en el aula a partir de un enfoque sociocultural buscando las interrelaciones que forman el triángulo interactivo de Coll y Solé (2001), que son la influencia educativa o ayuda prestada a la actividad constructiva del alumno en un proceso de ajuste constante. Encontraron que los episodios de interactividad de los salones que observaron compartían elementos en común, como la comunicación cara a cara bajo la pauta del docente en un inicio, pero con un paulatino traslado de la participación predominante a los alumnos. También identificaron monólogos docentes que a visión de los autores inhiben el desarrollo del pensamiento científico, diálogos triádicos que fueron predominantes en ambos cursos y que generan que el alumno aprenda a responder a estructuras de control docente tal y como Mehan (1979) y Lemke (1990) describieron, e intercambios de construcción conjunta que promovían la interacción con el conocimiento y se volvía un espacio para que no sólo el docente sino entre iguales hicieran intervenciones de ajuste.

Estudios tempranos en la interacción en el aula se enfocaron en descubrir patrones de discurso por medio de expresiones del docente; estos trabajos revelaron que el habla en el aula estaba estructurada (Sinclair y Coulthard, 1975), y por ello podía ser categorizada, cuantificada y analizada. Sinclair y Coulthard influyeron en demasía la lingüística social al proponer una estructura de interacción tríadica al concluir que había al menos un entendimiento tácito entre estudiantes y maestros acerca de quién habla y cuándo lo debe hacer. Esto abría paso a un intercambio muy estructurado consistente en una iniciación del docente (I), a manera de una pregunta, resultando en la respuesta del alumno $(R)$, que en turno era seguida por la retroalimentación por parte del docente (F). Esto llevó a que la secuencia IRF se volviera el punto de origen de diversas investigaciones, aun cuando esta estructura fuese nombrada de otras maneras: IRE por Mehan (1979), Diálogo triádico por Lemke (1990), o guión de recitación por Tharp y Gallimore (1989).

Acorde a Clarà y Mauri (2013), Coulthard y Brazil (1981) incorporaron otro principio explicativo que se basaba en la observación de cómo un movimiento del locutor predecía un movimiento de respuesta del interlocutor; años después, Wells (1999) denominó esto "Prospectividad" (Prospectiveness): un movimiento en el discurso 
que se presenta ante la obligación de proporcionarr una respuesta para el movimiento anterior se torna prospectivo. Al mismo tiempo, los distinguió como dependientes o independientes con base en si su espacio de inicio se encontraba en el espacio $\mathrm{R}$ o $\mathrm{F}$ de otro intercambio.

Hall y Walsh (2002) distinguieron entre la $E$ y la $F$ argumentado que los usuarios de la evaluación verían el proceso de aprendizaje como un proceso de transmisión, mientras que los que utilizaban la retroalimentación valoraban las actividades de exploración, prueba de hipótesis y resolución de problemas, lo que hace del aprendiz más activo en su propio aprendizaje, a la vez que muestra que el docente desea generar actitudes y aptitudes de un "profesional reflexivo.

Investigaciones recientes (Villalta-Páucar, Martinic-Valencia y Guzmán-Droguett, 2011) se han basado en la clasificación de las estructuras de intercambio de sala de clase según las demandas cognitivas (consideradas como el grado requerido de que el alumno reestructure conocimientos previos) que implicaban, esta clasificación fue elaborada originalmente por Wells y Mejía-Arauz (2005) y consiste en intercambios de baja exigencia cognitiva, como el transmitir conocimiento, es decir, de pregunta-respuesta, de validación, donde la tercera intervención es para aceptar la segunda, y co-formados donde la respuesta es inducida mediante pistas del profesor. Mientras estas estructuras de intercambio son memorísticas y el docente sólo brinda conocimiento, las de alta exigencia cognitiva implican al alumno en una situación activa donde depende de él la negociación de información, y se dividen en intercambios explicativos (donde se profundiza a detalle en elementos y detalles de las preguntas de los alumnos), cooperativos (donde la tercera parte sirve para contribuir y enriquecer la segunda aportación) y colaborativos (donde el intercambio de conocimiento es más simétrico y se expone a debate el saber que se está compartiendo).

Park (2020) desarrolló una investigación en el aula dividiendo la manera en que el docente elicitaba la respuesta del alumno en dos formas: el método abierto y el método limitante (opening up and limiting), consistentes en preguntas evaluativas hechas a todos los alumnos o alumnos en específico, encontrando que los docentes hacían preguntas de variable dificultad por medio del método abierto, a manera de una puesta a prueba con las expectativas que tenía del desempeño de los alumnos en la adquisición de conocimientos de la clase; esto lleva a elucidar la importancia del modo de preguntar del docente para la complejidad de respuesta del alumnado.

Los intercambios presentados muestran que las secuencias IRE se encontrarían en la categoría de valida- ción al ser de baja exigencia cognitiva y de estructura simple, lo que concuerda con Wells y Mejía-Arauz (2005) al definir como recitación memorística al intercambio inicio-respuesta-seguimiento (su traducción de IRE); asimismo, Stierer y Maybin (1994) describen el diálogo tríadico tradicional como inadecuado para el aprendizaje y expresión de conocimiento en el aula por parte de los alumnos, y colocan a las secuencias que involucran una retroalimentación dentro de las categorías de alta exigencia; sin embargo las retroalimentaciones donde el docente induzca la respuesta continuarían siendo de baja exigencia cognitiva al ser de naturaleza memorística, por lo que la información sugiere que una retroalimentación constante no necesariamente lleva a una co-construcción y reinterpretación del conocimiento por parte del alumno, sino sólo las que conducen a que el alumno reintegre sus conocimientos o los exponga para confrontar lo expuesto durante la clase.

Para entender la manera en que los participantes de esta actividad se involucran, se puede usar del análisis conversacional; para Waring (2011) su uso incrementa la atención a la naturaleza emergente y compleja de las interacciones del lenguaje; documenta cómo el aprendizaje ocurre a través del tiempo y tras la reconceptualización del aprendizaje como una participación en la teoría sociocultural sirve a su vez para obtener más información de las prácticas interaccionales que crean o inhiben las oportunidades para participación, y por extensión las oportunidades de aprendizaje.

Las investigaciones enfocadas en el lenguaje (Jacknick, 2011; Waring, 2011) han mostrado que hay ocasiones en las cuales el alumno pregunta información esperando ser contestada por el docente, situaciones que no corresponden a la secuencia IRE/F desde el momento en donde son iniciadas por el alumno, quienes, con habilidad y respetando la situación del docente como moderador y conocedor primario, buscan obtener información específica acerca de los temas vistos en clase. Estas iniciativas del aprendiz abren acceso a varias oportunidades de aprendizaje a partir de una acción casual, como lo es bromear o comentar a partir de algo vivido por ellos, apartada de un objetivo académico, como lo sería explicar o exponer (Waring, 2011).

De la misma manera, Waring (2011) indagó la agencia del aprendiz en la formación de oportunidades de aprendizaje, encontrando y dividiendo sus iniciativas de aprendizaje en tres bloques diferentes: 1) cuando el alumno inicia el turno; 2) cuando hay una autoselección donde el alumno tiene la iniciativa de responder, y 3) cuando se puede explotar un turno asignado para comenzar una secuencia; los resultados muestran 
que el alumno es capaz de estructurar oportunidades de aprendizaje mostrando su agencia; sin embargo hizo notar que es necesario encontrar un balance donde los alumnos sean capaces de contribuir más que afectar la agenda del docente durante sus iniciativas.

Li (2013) también se interesó por las iniciativas del estudiante al considerarlas como generadoras de contingencia en la secuencia IRF, y también capaces de crear oportunidades de aprendizaje. Fundando su investigación en los hallazgos de Waring (2011) buscaba cumplir dos propósitos: demostrar cómo el análisis de la conversación en general puede proporcionar luz en los momentos pedagógicamente significantes en la interacción del aula, e ilustrar cómo docentes de lenguas extranjeras y en particular docentes de lengua china en particular pueden beneficiarse de un microanálisis de sus prácticas y de las de otros. Waring (2011) muestra la premisa de que el fenómeno observado donde los aprendices al tomar iniciativas usan el lenguaje para configurar y reconfigurar patrones de participación y crear oportunidades de aprendizaje está relacionada con la visión sociocultural del aprendizaje del lenguaje como participación.

Por último, Waring, Reddington y Tardic (2016) abordaron el tema de la investigación de Waring (2011), en un artículo que describía dos prácticas docentes donde el control del docente se mantenía sin perder la participación y el aprendizaje, haciendo un recuento de investigaciones que se encargan de comprender cómo los docentes controlan aportes del alumno que podrían considerarse inesperadas o desordenantes.

De manera similar, Tronchoni (2019) indica que la para conseguir una cooperación dialógica a partir de varios actos comportamentales, y en el caso de las clases de tipo expositivo los actos de dar-tomar y pedir-recibir son los principales conductores dentro del flujo discursivo, mientras que el compartir conocimientos y experiencias en clase cumple la función de andamiaje necesario para que el alumno pueda avanzar a representaciones más complejas de conocimiento.

La investigación centrada en la enseñanza dentro de la carrera de Medicina en México (Lazcano, Valenzuela y Conget, 2013; Urrutia-Aguilar y Guevara-Guzmán, 2013) se ha dedicado al estudio de las estrategias del docente durante las clases y las variaciones de consecuencias que tienen en la interacción profesor-alumno dentro del salón de clases.

Lazcano, Valenzuela, y Conget (2013) desarrollaron un estudio orientado a conocer que teorías implícitas de la enseñanza tenían más predominancia en docentes de la carrera de Medicina, así como la manera en que vinculaban estas teorías a diferentes modelos pedagógicos. EI supuesto principal que los llevó a efectuar la investigación es que la práctica pedagógica está condicionada por las teorías implícitas de la enseñanza a las que adhieren los docentes, por lo que esta puede afectar de manera directa la implementación de las estrategias propuestas durante reformas educacionales. Los autores encontraron que los docentes se adhieren en su mayoría a la teoría expresiva e interpretativa al creer que el estudiante es el centro del proceso formativo; por ende, haciendo uso de modelos pedagógicos activos y constructivistas, sus teorías implícitas están condicionadas por sus vivencias como estudiantes y profesionales, que a la capacitación docente que han recibido mediante cursos o en el día a día. Esto implica que es durante la interacción en el aula que los docentes se han formado, y forman maneras de actuar como profesionales, ser en su rol de docentes, y aprender conocimientos y formas de interactuar con otros.

Por su parte, Urrutia-Aguilar y Guevara-Guzmán (2013) analizaron la relación entre la opinión de los estudiantes respecto a las estrategias de enseñanza utilizadas por el profesor en el aula y el aprovechamiento académico de los estudiantes; la intención surgió porque en la carrera de Médico Cirujano que se imparte en la Facultad de Medicina de la Universidad Nacional Autónoma de México (UNAM) se había reportado un alto índice de alumnos que no acreditaban el primer año de la carrera, sobre todo en las materias del área básica, lo que implicaba deserción y recursamiento en gran escala. Los investigadores encontraron que los docentes con mayor cantidad de estrategias docentes llevaban a un mejor aprovechamiento académico, por lo que concluyeron que el docente, como facilitador del aprendizaje, debe contar con suficiente capacitación didáctica que le permita implementar estrategias de enseñanza durante su práctica.

Finalmente, la manera en que el docente instruye y construye con el alumno, la manera en que se expresa y ve al otro, adquiere un papel muy relevante como producto de la interacción que se da entre docente y alumno. Coscia (2015) destaca la importancia del lenguaje como herramienta de mediación social, de comunicación y negociación en la interacción, y de mediación simbólica. El lenguaje permite dar sentido al cúmulo de construcciones, experiencias y actividades que se efectúan, en este caso, durante los procesos de enseñanza y de aprendizaje, y la manera en que el docente se conduce durante la clase define de manera determinante la forma de participación del alumno, su postura respecto a participar en compañía del docente, y la actitud que asumirá ante los conocimientos a adquirir y tareas a desarrollar durante su preparación profesional. 
Ávila-Morales (2017) hizo una investigación teórica respecto a los causantes de la deshumanización en el ejercicio profesional de medicina, interpretando deshumanización como la incapacidad de experimentar emociones humanas complejas, de compartir creencias o de actuar según los valores y las normas sociales y morales. Explica que en la enseñanza de Medicina hay factores relevantes dentro de la enseñanza de esta disciplina que llevan a actitudes deshumanizadas, como la observación de comportamientos poco éticos en los docentes que hagan uso de modelos de aprendizaje basados en la burla, el maltrato y la deshumanización.

Dado el amplio conocimiento de estos procesos en el aula, así como la disponibilidad de las publicaciones llenas de ideas para alterar el patrón de diálogo IRE (Waring, 2009, 2011; Jacknick, 2011), se esperaría que los profesores usaran un repertorio de diferentes estrategias a lo largo de su enseñanza; sin embargo Sert y Seedhouse (2011) argumentan que una gran cantidad de aprendizaje alrededor del mundo continúa dirigido por medio de formas tradicionales de enseñanza dominadas por la interacción con el docente al frente del aula y controlada por la naturaleza asimétrica de la distribución de turnos. Acorde a Nunn (2001), el diálogo triádico, más que ser sólo una estructura de discurso, es un ritual cotidiano en el contexto escolar que da orden y coordina la relación educativa similar a la interacción padre-hijo (Seedhouse, 2004), volviéndolo un acompañante del andamiaje en contextos educativos, junto con la estructura PE/F. Esto aunado a las observaciones de Villalta-Páucar y Martinic-Valencia (2013) al referir que la manera en que los docentes dan prioridad a diferentes competencias profesionales afecta de modo directo los tipos de intercambios comunicativos que tiene con el alumno, por lo que la estructura de diálogo puede variar por efecto directo de estas apreciaciones y la manera en que el docente y los alumnos construyen su identidad y rol dentro del aula.

Esto enriquece la postura constructivista de la presente investigación al reafirmar que en la manera con que se establece el discurso es como se da lugar a conflictos o coordinaciones que pueden afectar en tiempo real el proceso de enseñanza-aprendizaje, sobre todo en las situaciones donde el docente está al frente del aula, porque en estas condiciones tiene más capacidad de control en la interacción (Nassaji y Wells, 2000), razón por la cual es necesario generar mayor conocimiento respecto a los diversos elementos que comprenden el diálogo docente. Con esta investigación se pretende obtener información que pueda servir como material útil para el diseño de cursos de preparación docente, una necesidad que otros autores, como Urrutia-Aguilar y
Guevara-Guzmán (2013), han colocado sobre la mesa como una oportunidad de generar docentes de la carrera de Medicina mejor preparados y con mayor número de estrategias educativas que causen efecto en el aprendizaje de sus alumnos.

Pregunta de investigación: ¿Cuáles son las estrategias discursivas usadas por el docente durante la interacción con los alumnos en el aula durante las clases de Medicina, y cómo son percibidas por los participantes de esta interacción?

Objetivo: Identificar las estrategias discursivas practicadas por el docente durante su interacción con los alumnos, y la percepción del alumno con base en esta interacción, en clases teóricas de la carrera de Medicina en una IPES.

\section{MÉTodo}

\section{Participantes}

Los participantes fueron cinco profesores (una mujer y cuatro hombres) de la asignatura teórica "seminario de integración socio-psico-biológica I" correspondiente a la carrera de Medicina de una IPES, y los alumnos de sus grupos $(n=144)$, y corresponden a $42 \%$ del alumnado cursando esa asignatura. Los participantes fueron seleccionados mediante un muestreo de sujetos-tipo; los criterios de inclusión fueron: profesores con asignaturas teóricas correspondientes a la carrera de Medicina de IPES seleccionada y los alumnos correspondientes a una de sus clases. En la tabla 1 se muestra la distribución de los alumnos por grupo y semestre al que corresponden.

\section{Tabla 1.}

Estadística de los participantes por grupo.

Grupo Muestra Hombres Mujeres Edad Semestre

\begin{tabular}{cccccc|}
\hline A & 27 & 8 & 19 & $18-27$ & 3 \\
\hline B & 31 & 12 & 19 & $17-21$ & 1 \\
\hline C & 27 & 10 & 17 & $18-29$ & 3 \\
\hline D & 32 & 13 & 19 & $17-22$ & 1 \\
\hline E & 27 & 8 & 19 & $17-25$ & 1 \\
\hline Total & 144 & 51 & 93 & $17-29$ & $1-3$ \\
\hline
\end{tabular}

\section{Materiales}

Se utilizó una videocámara con micrófono externo, así como un protocolo de registro de interacciones discursivas y también el Inventario de Interacción Profesor-Alumno (IIP-A), el cual consiste en 31 ítems en escala tipo Likert; de cuatro opciones de respuesta (Totalmente 
de acuerdo, De acuerdo, En desacuerdo, Totalmente en desacuerdo), el cuestionario fue creado con la finalidad de conocer la opinión de los estudiantes respecto a su interacción con los docentes al impartir su clase (Ruiz, González-Celis, Cruz y Hernández, 2018), y en la investigación permitió observar el grado de acuerdo en general que los alumnos dan en relación con su interacción con el docente en cuatro dimensiones.

1. Estrategia metodológica (EM). Valoración del uso de recursos pedagógicos del docente, que incluyen el uso de actividades didácticas y de herramientas en el aula, que sirven para que los estudiantes alcancen los niveles de competencia que no podrían conseguir por sí solos.

2. Enseñanza colaborativa (EC). Valoración al docente acerca de su modo de comunicarse, la directividad de la clase, su rol orientador y/o facilitador, su nivel de control de la clase e imparcialidad en la evaluación de los estudiantes.

3. Oportunidad de participación (OP). Valoración de la manera en que el profesor propicia ocasiones de exposición, argumentación y discusión, promoviendo participación de los alumnos.

4. Apreciaciones positivas de la interacción (API). Opinión personal del alumno referente a la interacción que llevó con el docente.

\section{Consideraciones éticas}

El trabajo se desarrolló siguiendo las recomendaciones del código ético del psicólogo de la Sociedad Mexicana de Psicología (2010), que indican trabajar con el consentimiento informado de los participantes, guardar su confidencialidad al usar seudónimos para evitar la identificación de la persona, e informándoles de cuando se les iba a grabar, así como de su derecho a obtener la trascripción de los datos que fueron analizados.

\section{Procedimiento}

Después entrar en contacto con los participantes, explicarles los objetivos de investigación y procedimiento, y obtener el consentimiento informado para efectuar la observación, se ingresó al salón de clases de los participantes en tres ocasiones diferentes. Las sesiones de observación se desarrollaron durante la etapa final del ciclo escolar, debido a que era requerida una interacción entre docente y alumnos ya consolidada para promover significancia entre las secuencias discursivas en el salón de clases. Se hizo uso de la videocámara con micrófono externo colocándola dentro del salón donde el docente y los alumnos tomaban clase. Al concluir la última obser- vación, se aplicó el cuestionario IIP-A a los alumnos de cada grupo; la grabación fue transcrita a posteriori para poder usar categorías de análisis, y los datos recabados en el IIP-A fueron vaciados en el software SPSS22.

\section{Mediciones}

El instrumento de conversión de los datos observados en categorías de análisis; se trata de una combinación entre formato de campo y sistemas de categorías, generado por medio del establecimiento de un rango jerárquico de unidades de conducta (Anguera-Arguilaga, Santoyo y Espinoza, 2003) a partir de un marco teórico fundamentado (Anguera-Arguilaga e Izquierdo, 2006), que en este caso es la definición de los patrones IRE/F y PE/F, así como las conductas que se observen en las grabaciones y requieran su modificación ad hoc. Esto llevó a un sistema taxonómico de categorías de observación bien definidas, con unidades de conducta operacionales, validada mediante jueceo de expertos en el estudio del análisis del discurso en el aula.

Las unidades de conducta fueron hechas a partir de las tres condiciones sugeridas por Anguera-Arguilaga, Santoyo y Espinoza (2003), que consisten en ser identificables por sí mismas, denominables y definibles, buscando llegar a la exhaustividad y la mutua exclusividad (E/ME) de las mismas, para que exista una categoría para cada acción posible, y una acción posible para cada categoría de interés. Con la intención de identificar la estructura discursiva del hablante en turno, las características interactivas de la actividad se codificaron considerando las funciones sociales (habla, relación y tarea) que cumplen (Anguera e Izquierdo, 2006). Cada turno se codificó como un "todo" en el cual no se tiene en cuenta el tiempo de duración ni latencia entre turnos, ubicando cada uno como un evento temporal simple (Bakeman y Quera, 2011).

De esta manera la codificación generada con base en el marco teórico y las observaciones resulta en la taxonomía que se muestra en la tabla 2.

Cada una de las categorías planteadas fue vista con una probabilidad de ocurrencia presente en cualquier participante, distinguidos con base en su relación con el conocimiento expuesto (como aprendiz o como experto del mismo); con la finalidad de diferenciar el rol en el aula del participante que llevó a cabo la categoría, al comienzo de cada una de ellas se colocó una " $M$ " en caso de que haya sido presentada por el maestro, y una "A" cuando fuera alguno de los alumnos (por ejemplo, Maestro instruyendo explicando: MInsexp). 
Tabla 2.

Categorías de la secuencia discursiva, con clave y definición.

Elicitando: El profesor genera una frase con la intención de que la complete el alumno.

Inseli

Instruir: Acción donde un experto (poseedor del conocimiento) da información o conocimientos referentes a un tema a algún aprendiz (en proceso de conocer el(los) tema(s).

Pregunta moduladora: Acción donde un Experto pregunta información o conocimientos referentes a la actividad a algún aprendiz en búsqueda del saber que poseen al respecto.
Explicando: Exponer un saber para hacerlo más comprensible mediante el discurso.

Insexp

Orientando: Dirigir al otro hacia elementos del contexto necesarios para la actividad.

Guiando: Dirigir tareas referentes al tema.

Insgui

Ordenando: Demandar el cumplimiento de una tarea.

Insord

Evaluando a cualquier participante: Determinar conocimientos y/o aptitudes de cualquier miembro de un grupo.

Preeva

Evaluando a un participante en específico: Determinar conocimientos y/o aptitudes de alguien en particular.

Predeva

Sondeando: Conocer si existen dudas ante una explicación o retroalimentación.

Preson

Simple: Sin argumentar o entrar en detalles.

Presim

Preguntar: Acción donde un aprendiz pide información para obtener conocimientos relativos a las prácticas llevadas a cabo.

Ordenando: Generar una acción por parte del otro.

Preord

Orientando: Señalando elementos del contexto relativos a lo que se desconoce.

Preori

Explicando: Exponiendo a detalle las características de la interrogante. Preexp

Dudando: Usar un conocimiento que aún no está estructurado.

Redud

Ordenando: Demandar el cumplimiento de una tarea.

Reord

Confirmando: Corroborar información.

Reconf

Responder: Acción donde un aprendiz contesta ante una instrucción o pregunta mostrando el conocimiento adquirido.

Reinterpretando: Usar un conocimiento reconstruido a partir de lo enseñado.

Rereint

Simple: Sin argumentar o entrar en detalles.

Resim

Explicando: Exponer un saber para hacerlo más comprensible.

Reexp

Negando: Reconocer la falta de un saber propia.

Reneg

Afirmando: Reconocer la presencia de saber propia.

Reafi

Validando: Dando como válida la información mostrada por el otro.

Evaval

Rechazando: Dando como inválida la información mostrada por el otro.

Evarech

Evaluar: Acción donde el experto calcula
Calificando: Puntuar el nivel de aceptación del conocimiento del otro con base en un juicio de valor.
Evacal 
Parafraseando: Reinterpretar la respuesta dada por el estudiante con el objetivo de enseñarle nuevas estructuras de entendimiento.

Corrigiendo explícitamente: Proporcionar la respuesta correcta.

Fcorr

Feedback: Acción donde un experto proporciona información importante

F a los aprendices sobre sus conductas previas observadas y/o evaluadas, con la intención de que tenga oportunidades de aprendizaje y reflexión.

$\mathrm{Xn}$
Recast: reacoplar la oración del alumno a la esperada por el profesor. Frec

Explicando: Proporcionar la respuesta e información adicional respecto a la misma.

Fexp

Dando claves o pistas: Dar información sobre el error del estudiante, por medio de claves léxicas que él debe relacionar para alcanzar sentido y continuar con el discurso.

Fclue

Elicitando: El profesor genera una pregunta o frase con la intención de que la complete el alumno.

Feli

Comentario: Contribución espontánea y personal respecto a la acción previa del participante.

$X A$

Escucha activa: Comentarios monosilábicos donde el participante corrobora el mensaje del otro sin tomar turno (ej. Aham, uhumm, entiendo).

$\mathrm{XB}$

Tiempo de espera prolongado: El participante sólo agrega aclaraciones a los argumentos del otro, sin tomar control del tema de conversación XC en curso.

Asignar turno de participación: El participante da el turno a quien desee hablar.

$X D$

\section{AnÁlisis estadísticos}

Para codificar las transcripciones se usaron las categorías de análisis (tabla 2) en cada acción discursiva hecha por los participantes, generando una agrupación secuencial codificada, que se volvió la base de datos. Se utilizó la técnica de análisis estructural, traduciendo al lenguaje SDIS (Sequential Data Interchange Standard) para poder aplicar el programa de análisis secuencial GSEQ (Generalized Sequential Event Querier) (Bakeman y Quera, 1996). EI SDIS-GSEQ se apoya en una técnica analítica desarrollada por Bakeman (1978), en colaboración con Gottman (Bakeman y Gottman, 1986) y por Sackett $(1980,1987)$, que siguen siendo de gran relevancia (Bakeman y Quera, 2011) para analizar datos secuenciales $y$, en esta investigación, sirve para construir los patrones IRE/F y PE/F efectuados durante el proceso de enseñanza-aprendizaje al analizar los patrones secuenciales de discurso, así como las diferencias en su predominancia que tienen durante la clase.

Para identificar los patrones estadísticamente significativos en el flujo de discurso se aplicaron las técnicas de análisis secuencial, que son las pausas o distancia temporal entre la aparición de los elementos observados, en nuestro caso de los actos de habla, llevados a cabo con la búsqueda de contingencias secuenciales entre las categorías de análisis. La investigación se enfoca en las relaciones secuenciales en el discurso de los participantes de habla identificados, considerando los que tengan residuales ajustados con un valor $Z$ superior a 1.96 (valor $p<0.05$ ) y un coeficiente de correlación Phi por encima de 0.20 (valor $p<0.05$ ). Este tipo de análisis sirve para la detección de patrones secuenciales de conducta (Quera, 1993), lo cual se logra buscando contingencias secuenciales entre las categorías de análisis, en nuestro caso, relaciones secuenciales en el discurso de los participantes, teorizadas a partir del principio de prospectividad de los pares adyacentes.

De la misma manera, para analizar los datos del cuestionario IIP-A los datos vaciados en el software SPSS22 fueron integrados en sus dimensiones correspondientes, llevando a cabo una alpha de Cronbach en cada grupo para confirmar la consistencia interna entre los ítems de la prueba; después los valores por dimensión fueron convertidos en porcentajes para de esa manera observar el grado de acuerdo que los alumnos tenían hacia el desempeño del docente en la dimensión evaluada. 
Tabla 3.

Porcentajes de acuerdo con la interacción en el aula obtenidos por medio del IIP-A.

\begin{tabular}{|c|c|c|c|c|c|c|}
\hline \multirow{2}{*}{ GRUPO } & \multicolumn{6}{|c|}{$\begin{array}{l}\text { Porcentaje de acuerdo con los factores del Inventario de Interacción Profesor- } \\
\text { Alumno (IIP-A) }\end{array}$} \\
\hline & $\begin{array}{l}\text { ESTRATEGIA } \\
\text { METODOLÓGICA }\end{array}$ & $\begin{array}{l}\text { ENSEÑANZA } \\
\text { COLABORATIVA }\end{array}$ & $\begin{array}{l}\text { OPORTUNIDAD } \\
\text { DE } \\
\text { PARTICIPACIÓN }\end{array}$ & $\begin{array}{l}\text { APRECIACIONES } \\
\text { POSITIVAS DE } \\
\text { LA INTERACCIÓN }\end{array}$ & $\begin{array}{l}\text { CALIFICACIÓN } \\
\text { TOTAL IIP-A }\end{array}$ & $\begin{array}{l}\text { AlPha DE } \\
\text { Cronbach }\end{array}$ \\
\hline A & 83.00 & 84.58 & 85.47 & 84.37 & 84.47 & 0.953 \\
\hline B & 90.50 & 91.66 & 89.52 & 91.12 & 90.45 & 0.859 \\
\hline C & 72.75 & 86.08 & 71.40 & 72.68 & 73.60 & 0.836 \\
\hline D & 78.91 & 81.00 & 75.95 & 76.28 & 77.41 & 0.937 \\
\hline$E$ & 94.75 & 96.00 & 94.45 & 96.06 & 95.11 & 0.898 \\
\hline $\begin{array}{l}\text { Muestra } \\
\text { completa }\end{array}$ & 84.00 & 86.00 & 83.27 & 83.18 & 83.98 & 0.962 \\
\hline
\end{tabular}

\section{Resultados}

\section{Estadística descriptiva del IIP-A en la muestra completa}

En la tabla 3 se muestran las estadísticas descriptivas de los resultados del IIP-A en todos los grupos registrados.

Se observa que la calificación promedio de los grupos fue de $83.98 \%$ de acuerdo con la interacción con el docente en el salón de clases; los grupos con porcentajes generales de acuerdo por encima de $90 \%$ fueron el B $(90.45 \%)$ y el E $(95.11 \%)$, mientras que los grupos C $(73.60 \%)$ y D $(77.41 \%)$ tuvieron porcentajes de acuerdo con la interacción en el aula menores a $80 \%$. El alfa de Cronbach de cada una de las aplicaciones del instrumento (una por grupo) se ubicaron entre 0.898 y 0.953 , representando buena consistencia interna entre los ítems de la prueba.

\section{Frecuencia de eventos registrados de la muestra completa}

Para esta investigación un total de 30 horas de grabación fueron recabadas, trascritas y codificadas, identificando 4,760 eventos de interacción en el aula, con un mínimo de 536 y un máximo de 1,236 eventos por grupo, cuyas frecuencias se presentan en la tabla 4.

Se puede observar que los eventos principales registrados fueron el Alumno instruyendo explicando (como expositor, Alnsexp), Respondiendo explicando (AReexp) y Respondiendo sin entrar en detalles (AResim), y en el caso del docente: Instruyendo explicando (MInsexp), Preguntando si existían dudas por aclarar (MPreson), Validando la aportación del otro (MEvaval),
Retroalimentando a detalle (MFexp), y Haciendo uso de comentarios personales (MXA).

\section{Correlación de categorías de discurso}

En la figura 1 se muestra el diagrama de flujo de los intercambios discursivos del grupo A en su primer nivel categorial (instrucción, pregunta moduladora, respuesta, evaluación, retroalimentación, pregunta, y follow-up); en el esquema se puede observar que las preguntas moduladoras del maestro y alumno, así como las preguntas del maestro en papel de aprendiz llevaron a que el alumno respondiera, y de la misma manera que el docente, en rol de experto, retroalimente o evalúe; en el caso de que haya evaluado la respuesta del alumno, el docente reinició las secuencias IRE por medio de una instrucción. Por otra parte, el alumno ha logrado iniciar seguimientos que son continuados de la misma manera por el docente.

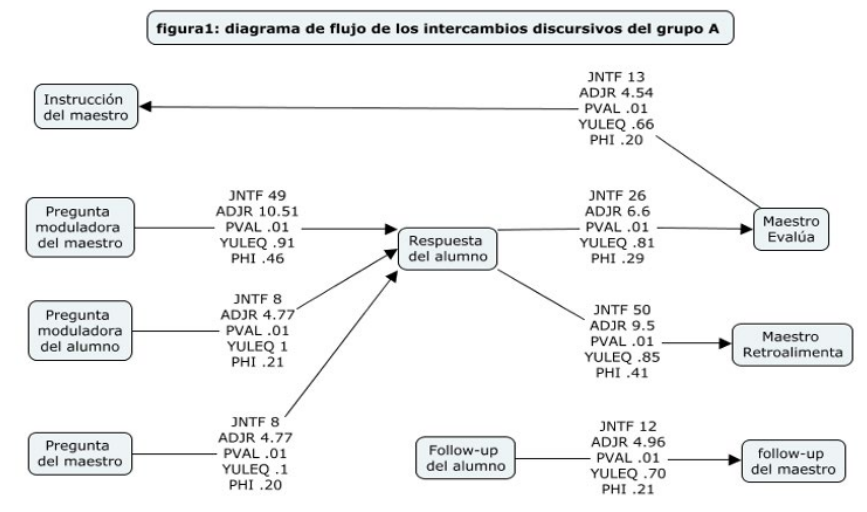

Figura 1

Diagrama de flujo de los intercambios discursivos del grupo A. 
Artículo Teórico | Estrategias discursivas y opinión del alumnado... Cruz-González \&Z Ruíz Carrillo

Tabla 4.

Frecuencia de eventos registrados por grupo.

\begin{tabular}{|c|c|c|c|c|c|c|c|}
\hline $\begin{array}{l}\text { Categoría } \\
\text { PRINCIPAL }\end{array}$ & Subcategoría & Grupo A & GRUPO B & Grupo C & GRUPO D & GRUPO E & TOTAL \\
\hline \multirow{3}{*}{$\begin{array}{l}\text { Instrucción del } \\
\text { alumno }\end{array}$} & Alnsexp & 41 & 9 & 22 & 85 & 90 & 247 \\
\hline & Alnsgui & 4 & 1 & 0 & 1 & 5 & 11 \\
\hline & Alnsord & 2 & 0 & 1 & 0 & 1 & 4 \\
\hline \multirow{4}{*}{$\begin{array}{l}\text { Instrucción del } \\
\text { maestro }\end{array}$} & Mlnsexp & 21 & 71 & 40 & 96 & 23 & 251 \\
\hline & MInsgui & 14 & 18 & 9 & 16 & 8 & 65 \\
\hline & MInsord & 33 & 26 & 47 & 54 & 21 & 181 \\
\hline & MInsori & 1 & 0 & 0 & 0 & 0 & 1 \\
\hline \multirow{2}{*}{$\begin{array}{l}\text { Pregunta } \\
\text { moduladora } \\
\text { del alumno }\end{array}$} & APreeva & 6 & 0 & 0 & 0 & 1 & 7 \\
\hline & APreson & 2 & 0 & 1 & 1 & 10 & 14 \\
\hline \multirow{2}{*}{$\begin{array}{l}\text { Pregunta } \\
\text { moduladora } \\
\text { del maestro }\end{array}$} & MPreeva & 28 & 35 & 14 & 91 & 10 & 178 \\
\hline & MPreson & 31 & 142 & 113 & 93 & 70 & 449 \\
\hline \multirow{4}{*}{$\begin{array}{l}\text { Pregunta del } \\
\text { alumno }\end{array}$} & APreexp & 2 & 4 & 6 & 4 & 0 & 16 \\
\hline & APreord & 1 & 0 & 1 & 1 & 0 & 3 \\
\hline & APreori & 0 & 1 & 1 & 0 & 0 & 2 \\
\hline & APresim & 5 & 9 & 14 & 5 & 15 & 48 \\
\hline \multirow{4}{*}{$\begin{array}{l}\text { Pregunta del } \\
\text { maestro }\end{array}$} & MPreexp & 0 & 2 & 2 & 0 & 0 & 4 \\
\hline & MPreord & 0 & 1 & 0 & 0 & 0 & 1 \\
\hline & MPreori & 0 & 0 & 1 & 0 & 0 & 1 \\
\hline & MPresim & 8 & 15 & 25 & 4 & 27 & 79 \\
\hline \multirow{9}{*}{$\begin{array}{l}\text { Respuesta del } \\
\text { alumno }\end{array}$} & AReafi & 11 & 18 & 38 & 26 & 22 & 115 \\
\hline & AReconf & 1 & 5 & 14 & 11 & 9 & 40 \\
\hline & ARedud & 8 & 28 & 8 & 26 & 7 & 77 \\
\hline & AReexp & 58 & 125 & 139 & 87 & 78 & 487 \\
\hline & AReneg & 1 & 2 & 7 & 1 & 2 & 13 \\
\hline & AReord & 0 & 1 & 0 & 0 & 0 & 1 \\
\hline & AReori & 0 & 3 & 7 & 1 & 0 & 11 \\
\hline & ARereint & 4 & 3 & 10 & 8 & 25 & 50 \\
\hline & AResim & 59 & 234 & 82 & 144 & 61 & 580 \\
\hline \multirow{4}{*}{$\begin{array}{l}\text { Respuesta del } \\
\text { maestro }\end{array}$} & MReafi & 0 & 1 & 3 & 0 & 0 & 4 \\
\hline & MReconf & 1 & 3 & 5 & 2 & 0 & 11 \\
\hline & MReexp & 0 & 2 & 1 & 0 & 1 & 4 \\
\hline & MResim & 0 & 1 & 3 & 0 & 1 & 5 \\
\hline $\begin{array}{l}\text { Alumno } \\
\text { evalúa }\end{array}$ & AEvaval & 2 & 0 & 0 & 0 & 0 & 2 \\
\hline
\end{tabular}




\begin{tabular}{|c|c|c|c|c|c|c|c|}
\hline $\begin{array}{l}\text { CATEgoría } \\
\text { PRINCIPAL }\end{array}$ & SubCATEgoría & Grupo A & GRUPO B & Grupo C & Grupo D & Grupo E & TOTAL \\
\hline \multirow{3}{*}{$\begin{array}{l}\text { Maestro } \\
\text { evalúa }\end{array}$} & MEvacal & 0 & 0 & 1 & 0 & 0 & 1 \\
\hline & MEvarech & 4 & 14 & 15 & 17 & 2 & 52 \\
\hline & MEvaval & 31 & 116 & 49 & 56 & 49 & 301 \\
\hline \multirow{3}{*}{$\begin{array}{l}\text { Alumno } \\
\text { retroalimenta }\end{array}$} & AFcorr & 0 & 1 & 0 & 0 & 1 & 2 \\
\hline & AFeli & 2 & 0 & 0 & 0 & 1 & 3 \\
\hline & AFexp & 1 & 0 & 2 & 0 & 3 & 6 \\
\hline \multirow{5}{*}{$\begin{array}{l}\text { Maestro } \\
\text { retroalimenta }\end{array}$} & MFclue & 4 & 8 & 4 & 20 & 0 & 36 \\
\hline & MFcorr & 3 & 20 & 33 & 22 & 15 & 93 \\
\hline & MFeli & 14 & 52 & 9 & 79 & 23 & 177 \\
\hline & MFexp & 44 & 92 & 87 & 65 & 58 & 346 \\
\hline & MFpar & 2 & 7 & 5 & 6 & 7 & 27 \\
\hline \multirow{4}{*}{$\begin{array}{l}\text { Follow-up del } \\
\text { alumno }\end{array}$} & AXA & 38 & 10 & 52 & 17 & 26 & 143 \\
\hline & $A \times B$ & 0 & 0 & 3 & 0 & 0 & 3 \\
\hline & $A \times C$ & 0 & 0 & 0 & 0 & 5 & 5 \\
\hline & $A X D$ & 0 & 0 & 3 & 1 & 3 & 7 \\
\hline \multirow{4}{*}{$\begin{array}{l}\text { Follow-up del } \\
\text { maestro }\end{array}$} & MXA & 38 & 75 & 133 & 40 & 53 & 339 \\
\hline & $M \times B$ & 0 & 19 & 8 & 1 & 0 & 28 \\
\hline & $M \times C$ & 5 & 10 & 10 & 17 & 15 & 57 \\
\hline & & 6 & 52 & 38 & 60 & 16 & 172 \\
\hline \multicolumn{2}{|c|}{ Total } & 536 & 1236 & 1066 & 1158 & 764 & 4760 \\
\hline
\end{tabular}

Los intercambios discursivos del grupo B (figura 2) mostraron que el maestro ha instruido y de inmediato ha hecho preguntas moduladoras para elicitar la respuesta del alumno, o ha ocupado follow-ups para la misma consecuencia. Tanto las preguntas como las respuestas del alumno han suscitado que el maestro retroalimente, pero las respuestas del alumno también pudieron ser evaluadas Ilanamente.

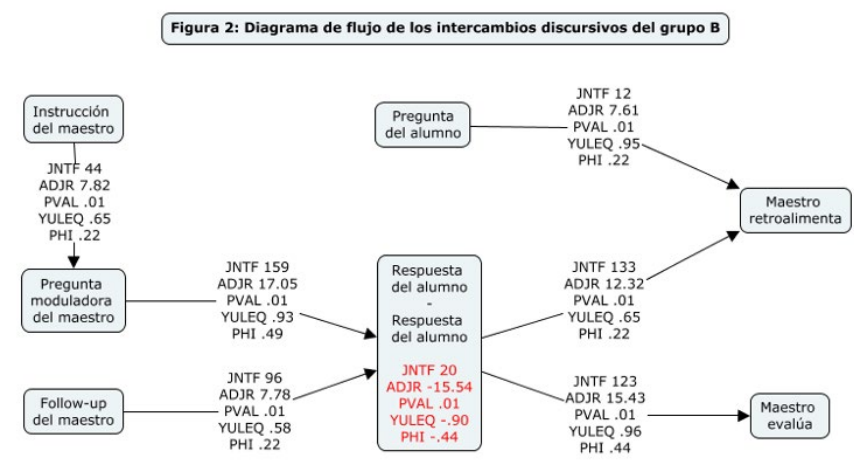

Figura 2

Diagrama de flujo de los intercambios discursivos del grupo $B$.
En el diagrama de flujo de los intercambios discursivos del grupo $\mathrm{C}$ en el primer nivel categorial (figura 3) se observa una secuencia IRE/F donde el docente ha hecho preguntas moduladoras para elicitar la respuesta del alumno, donde estas respuestas han sido evaluadas o retroalimentadas; en otro flujo diferente, al alumno instruyendo de modo recurrente sin correlación con otra categoría, muestra de una falta de cohesión/unión en el flujo de discurso.

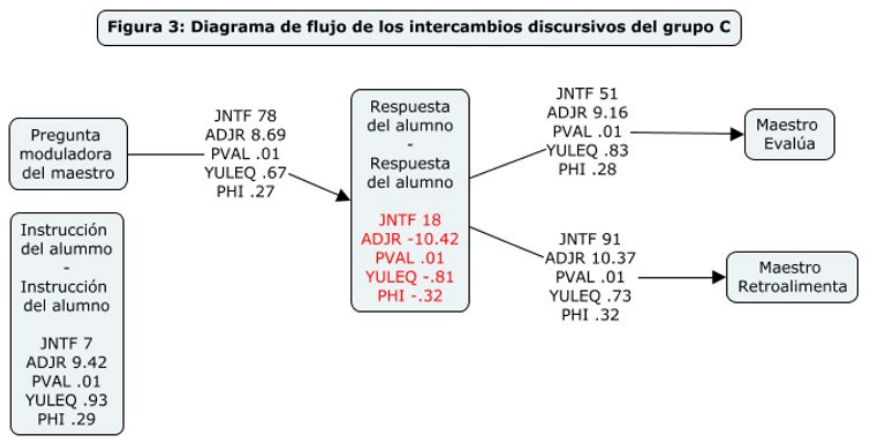

Figura 3

Diagrama de flujo de los intercambios discursivos del grupo C. 
En la figura 4 se muestra el diagrama de flujo correspondiente al grupo D; se pueden observar dos flujos de discurso: uno donde la instrucción docente ha contribuido a que el alumno participe instruyendo a sus compañeros, o a que el profesor generara preguntas moduladoras, y otro donde el follow up del docente elicitaba de manera significativa las respuestas del alumno, y ante esta respuesta el docente, al retroalimentar, ha generado ciclos donde tanto la retroalimentación docente como respuesta del alumno se tornaron recurrentes; también hay una correlación significativa entre la respuesta del alumno y la evaluación de la respuesta por parte del docente, mostrando que la retroalimentación del maestro ha sido efectiva y se vuelve un puente para que los alumnos participen de manera activa en el discurso de la clase.

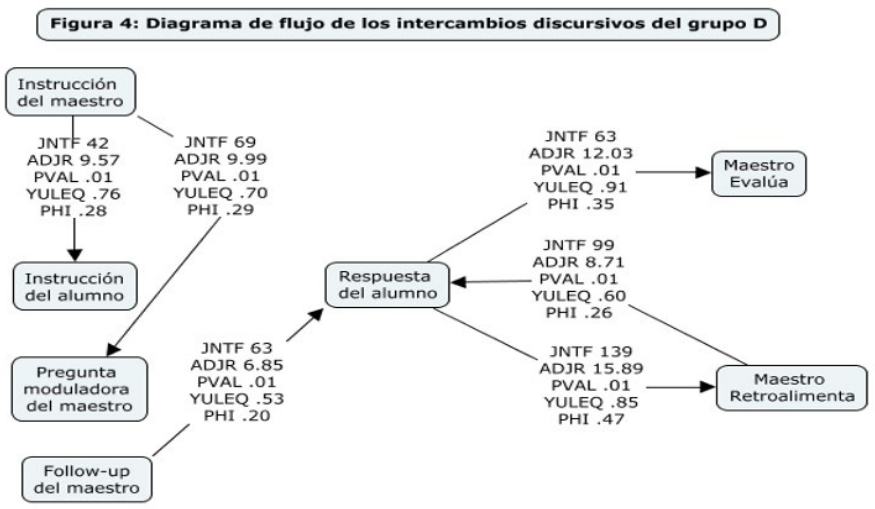

Figura 4

Diagrama de flujo de los intercambios discursivos del grupo $D$.

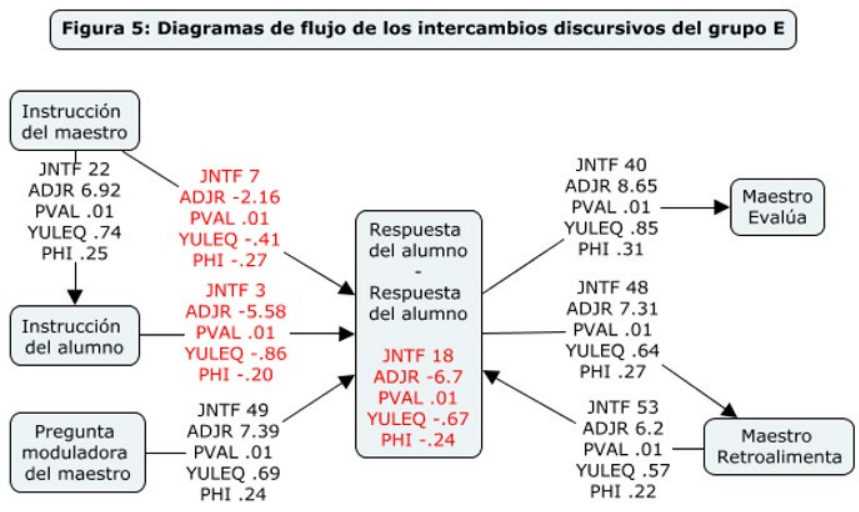

Figura 5

Diagrama de flujo de los intercambios discursivos del grupo $E$.

En el diagrama de flujo del Grupo E (figura 5) se muestra una correlación significativa y positiva entre la instrucción del docente y la posterior instrucción del alumno, y una correlación significativa y negativa entre la aparición de ambas instrucciones y la respuesta del alumno. Es por medio de que el docente hiciera preguntas moduladoras que se ha elicitado la respuesta del alumnado, y de modo similar al grupo D, el docente, desoués de retroalimentar, ha motivado la respuesta del alumno de manera recurrente; en caso de que el docente evaluara la respuesta, esto no ha conducido a otra categoría significativa.

\section{Discusión}

La investigación buscó identificar los efectos de las estrategias discursivas desarrolladas durante las clases de Medicina de una IPES, describiendo en el proceso las estrategias de intercambio discursivo (en forma de las diferentes configuraciones en las que docente y alumno intervienen, preguntan, responden, retroalimentan o evalúan entre ellos) y comparando estos resultados con la valoración que los alumnos tienen de esa interacción con el docente en la clase.

A partir de la tabla de frecuencia de eventos registrados por grupo se puede observar que los eventos con mayor ocurrencia corresponden a estructuras IRE/F; el docente ha llevado a cabo instrucciones, evaluaciones y retroalimentaciones, y comentarios personales complementarios; por otro lado, el alumno también ha sido capaz de instruir a manera de expositor. En los cinco grupos de Medicina que formaron parte de la investigación, el alumno no mostró su propia agencia (se reportó una baja presencia de preguntas por parte del alumno hacia el docente en búsqueda de información). En cuanto a interacciones, la mayor parte se fundó en una jerarquía de conocimiento en la cual el docente asumió un papel de experto y el alumno uno de aprendiz.

Mientras que Tronchoni, (2019) indicó que en el salón de clases predomina el carácter tradicional a partir del esquema "el profesor habla y los estudiantes escuchan" (p. 172), Lazcano, Valenzuela y Conget (2013) reportaron que los docentes de Medicina se adhieren a modelos pedagógicos y constructivistas; de los cinco grupos que formaron parte de la investigación, dos de ellos desarrollaron modelos tradicionales donde el docente estaba frente al aula, mientras que los tres restantes efectuaron interacciones donde el alumno asumía un papel más activo al instruir a sus compañeros como expositores.

Estas situaciones donde los alumnos han ocupado el papel de expositor frente al aula, instruyendo a sus compañeros, puede ser interpretado a partir de la teoría de la actividad de Engestrom (1993, citado en Lin, 2007) e implica que el alumno que expone tiene una posición diferente al alumno que está atendiendo a la exposición; por ende cambia la forma en que se llevan a cabo los intercambios comunicativos porque el expositor tiene conocimientos 
que el otro no. En esta situación la relación entre alumnos ya no es equivalente por jerarquía de conocimiento y genera una interacción donde se establece un andamiaje entre "iguales". Se podría esperar que ante la mayor similitud entre los agentes involucrados (ambos alumnos) se abrieran nuevas oportunidades de reestructurar la interacción, pero los alumnos continuaban el patrón IRE/F como si el rol del expositor no afectara la interacción discursiva.

Una explicación probable viene de los sub-roles que desempeña un docente y no lograron transmitirse al expositor; los alumnos que exponían podían abarcar el sub-rol definido por Hargreaves (1977, citado en Coll y Miras, 1993) correspondiente al de instructor, pero los expositores continuaban siendo dirigidos por el docente; aún tenía control completo del rol de manager o facilitador del salón, por lo cual la estructura de interacción era decidida por él de la misma manera en que lo haría sin ceder el lugar al frente del aula; los alumnos siguieron escuchando y el docente hablando al dirigir al alumno expositor; una acción muy negativa porque al mantener a los alumnos en el rol de oyentes se volvió ineficaz el proceso de enseñanza para hacer una construcción de significados compartidos (Tronchoni, 2019).

De hecho, en los cinco grupos se pudo observar que la secuencia IRE/F es el hilo principal con el cual se entrelaza el discurso escolar; la respuesta del alumno era retroalimentada o evaluada, y ésta se generaba después de una instrucción o secuencia moduladora. Sin embargo, los docentes de los grupos C y D obtuvieron porcentajes de acuerdo con la interacción por parte de sus alumnos de menores de $80 \%$, mientras que los docentes de los grupos B y E mayores a $90 \%$.

Al observar los diagramas de flujo discursivo se ve que los grupos $\mathrm{D}$ y $\mathrm{E}$, y $\mathrm{B}$ y $\mathrm{C}$ tienen semejanzas entre sí, pese a que el porcentaje de acuerdo obtenido en el IIP-A fue divergente. Los grupos D y E han sido capaces de llevar a mas allá la retroalimentación, generando bucles de discurso donde el alumno ha podido mantenerse respondiendo y ahondando en los temas y aprendizajes objetivo en la clase, mientras que en los grupos B y C la pregunta moduladora del docente ha llevado a que el alumno respondiera y en la mayoría de las ocasiones el docente retroalimentara estas respuestas.

El grupo A es el punto intermedio entre todos los grupos, y como tal se puede ver que comparte un poco de todos los grupos: las preguntas moduladoras de docentes y alumnos, así como las de tipo informal del docente Ilevan a que el alumno responda, y estas respuestas son su mayoría retroalimentadas, pero en caso de ser evaluadas el docente aprovecha la evaluación para reanudar la instrucción. Se puede observar también que tiene un bloque separado donde los seguimientos del alumno son respondidos del misma modo por el docente, llevando la situación en el aula a una plática informal.

Por último, los grupos $\mathrm{E}$ y $\mathrm{C}$ se alejaban de un control total dentro del salón de clases al observarse instrucciones de los alumnos como expositores; esto involucra los resultados de Lazcano, Valenzuela y Conget (2013), quienes encontraron a docentes que se adherían a modelos pedagógicos activos y constructivistas, y abrían espacios donde el alumno pudiera mostrar su propia agencia al iniciar turnos de participación, autoselección y explotación de sus turnos participativos (Waring, 2011), o en los raros casos de la investigación de Tronchoni (2019), en donde al generar una interacción participativa de alteridad (hablar para el otro) y colaboración (hablar a favor del otro) Ilevaban a dinámicas más enriquecedoras para los alumnos. En este caso, estos grupos adoptaban roles divergentes de los establecidos tradicionalmente.

El grupo A es el punto intermedio entre todos los grupos, y se pueden observar un cúmulo de semejanzas: las preguntas moduladoras de docentes y alumnos, así como las de tipo informal del docente, han llevado a que el alumno respondiera, y estas respuestas han sido en su mayoría retroalimentadas; en caso de ser evaluadas, el docente ha aprovechado la evaluación para reanudar la instrucción. También se puede ver que tiene un bloque separado donde los seguimientos del alumno han sido respondidos con más seguimientos por el docente, llevando la situación en el aula a una plática informal.

Los docentes de los grupos A, B y D han buscado mantener una posición de conocedores primarios, lo que a partir de los hallazgos de Gutiérrez-Giraldo y López-Isaza (2011) podrían generar episodios monológicos donde se podría inhibir el aprendizaje. Sin embargo, la ejecución de los docentes en los grupos A, E y B ha sido mucho mejor recibida por los alumnos que los de los grupos $C$ y $D$, aun si sus modelos pedagógicos eran distintos (el grupo $\mathrm{C}$ tiene un modelo abierto a que el alumno tome control de la clase, mientras que en el grupo $\mathrm{D}$ el docente se presenta constantemente frente al aula).

Se puede observar en los dos grupos con porcentajes de acuerdo menores a $80 \%$ flujos de discurso divididos en dos segmentos que no entran en contacto uno con el otro: en el caso del grupo $C$ se ha mostrado la secuencia IRE/F ya descrita, pero de modo adicional se ha encontrado al alumno instruyendo al alumno de manera recurrente, manteniendo una situación expositiva, mientras que en el grupo D el follow-up del docente ha llevado a respuestas del alumno que de ser retroalimentadas dirigieron a bucles más enriquecedores de la temática a tratar; pero también se encuentra otro bloque donde el maestro ha instruido y ge- 
nerado preguntas moduladoras que no llevaron de manera clara a otra categoría de interacción, sino que el alumno empezó a asumir el papel de expositor.

Esto implica una ruptura en la interacción en el salón de clases donde el flujo de discurso pierde cohesión, eventos que van de la mano con los resultados de Urrutia-Aguilar y Guevara-Guzmán (2013): mientras que los autores registraron que los profesores con mayor cantidad de estrategias docentes llevaban a un mejor aprovechamiento académico, en este estudio los alumnos identificaron las carencias en las estrategias docentes que pudieran generar un flujo de discurso constante y estable, afín para un ambiente positivo de interacción en el aula, es decir, la forma y el tipo de participación de los estudiantes así como su actitud frente a la información y a las tareas a efectuar durante el proceso de aprendizaje han sido condicionados tanto por la manera de organizar las actividades como el tipo de discurso de los docentes (Coscia, 2015).

Aun en una dinámica estrechamente centrada en el docente como conocedor primario el alumno ha tenido opiniones positivas, mientras el docente mantuviera cohesión en el discurso en compañía de los alumnos y no se enfocará en sólo mantener un diálogo con quien se encuentra en papel de expositor; esto ha llevado a una mayor motivación por parte del alumno a enfocarse en el discurso y conocimientos que eran compartidos dentro del salón de clases.

\section{Conclusiones}

Los resultados sugieren que el uso de estrategias metodológicas del docente dentro del salón de clase afecta la interacción profesor-alumno, el alumno se muestra sensible a la estrategia metodológica y la manera en que el docente desempeña su rol, y ubica al alumno dentro de un proceso de co-construcción de conocimiento que puede ser o no de su agrado, afectando la manera en que pueda negociar y co-construir significados en compañía de expertos e iguales.

Se coincide con la opinión de Jara-Gutiérrez, DíazLópez y Zapata-Castañeda (2015), quienes afirman que los modelos de evaluación formativa deben estar sustentados por el concepto de calidad docente y el perfil de un buen profesor de la facultad de Medicina, incluyendo el contexto en que trabaja, para que los estándares de evaluación se lleven a cabo tanto cualitativa como cuantitativamente en un ambiente de confianza.

Para esto es necesario promover la retroalimentación efectiva, hacer ajustes con base en los resultados, reconocer la profundidad de la evaluación y fomentar la capacidad de autoevaluarse y entender cómo mejorar; es indispensable que la institución educativa ofrezca al pro- fesor todos los escenarios requeridos para la formación, actualización e implementación de los cambios.

Si se acompaña la evaluación del docente con evaluaciones formativas mediante instrumentos sensibles a la práctica dentro del salón de clases como el IIP-A, puede abrir espacios de reflexión y de mejor comprensión de su actuar en el salón de clases; en otras palabras, incentivando el cambio a través de la autoevaluación. El verdadero desarrollo de la educación médica se logra en un entorno de aprendizaje y de reflexión individual crítica del profesor, donde éste sea capaz de hacer autoevaluaciones formativas. Esto le permite ser parte activa del proceso y, a la vez, reflexionar sobre lo que favorece o dificulta su propia labor; de esta manera identificará sus fortalezas y debilidades para lograr mejores resultados en el proceso de enseñanza-aprendizaje al fortalecer o cambiar sus acciones (Jara-Gutiérrez, Díaz-López y Zapata-Castañeda, 2015).

Para futuras indagaciones es relevante enmarcar y vincular las implicaciones que la historia del docente, su trayectoria y sus ideales ocupan en la manera en que elabora su discurso, siendo éste un elemento que acompaña de manera íntima la estrategia metodológica que ocupa, así como su modo de ver al alumno y su proceder para proporcionar y construir conocimiento al lado suyo; este elemento que no se retomó en esta investigación ayudaría a crear criterios que apoyaran al docente en la labor autorreflexiva que es necesaria para que modifique y mejore su enseñanza.

\section{Referencias}

Anguera-Argilaga, M. T., \& Izquierdo, C. (2006). Methodological approaches in human communication. From complexity of situation to data analysis. En G. Riva, M. T. Anguera, H. Wiederhold \& F. Mantovani (Eds.), From Communication to Presence: Cognition, Emotions and Culture towards the Ultimate Communicative. Amsterdam: IOS Press. http://dx.doi.org/10.1162/pres.16.5.559.

Anguera-Argilaga, M. T., Santoyo, C.. \& Espinosa, M. C. (2003). Evaluating links intensity in social networks in a school context through observational designs. En R. García Mira, J. M. Sabucedo Cameselle \& J. Romay Martínez (Eds.), Culture, Environmental Action and Sustainability (pp. 286298). Göttingen: Hogrefe \& Huber.

Argos, J., Ezquerra, P., Osorio, J. M., Salvador, L., \& Castro, A. (2013). La evaluación de los aprendizajes de los estudiantes en el marco del Espacio Europeo de Educación Superior (EEES): Sus prácticas, preferencias y evolución. European Journal of Investigation in Health, Psychology and Education, 3(3), 181-194.

Ávila-Morales, J. C. (2017). La deshumanización en medicina. Desde la formación al ejercicio profesional. Iatreia, 30(2), 216-229.

Bakeman, R. (1978). Untangling streams of behavior: Sequential analysis of observation data. En G. P. Sackett (Ed.), Observing Behavior (vol. 2): Data collection and analysis methods (pp. 63-78). Baltimore: University of Park Press.

Bakeman, R., \& Gottman, J. M. (1986). Observación de la interacción. 
Introducción al análisis secuencial. Madrid: Morata.

Bakeman, R., \& Quera, V. (1996). Análisis de la interacción: Análisis secuencial con SDIS y GSEQ. Madrid: Ra-Ma.

Bakeman, R., \& Quera, V. (2011). Sequential analysis and observational methods for the behavioral sciences. Cambridge: Cambridge University Press. http://dx.doi. org/10.1017/cbo9781139017343.

Clarà, M., \& Mauri, T. (2013). How Triadic Dialogue Allows Transfer of Responsibility in Dyadic Tutoring. Human Development, 56(5), 325-340.

Coll, C., \& Miras, M. (1993). Un punto de partida para el aprendizaje de nuevos contenidos: Los conocimientos previos. El constructivismo en el aula. Barcelona: Graó, 47-63.

Coll, C., \& Solé, I. (2001). Enseñar y aprender en el contexto del aula. En C. Coll, J. Palacios y A. Marchesi (comps.). Desarrollo Psicológico y Educación, 2.

Coscia, P. (2015). Las estructuras de participación de los estudiantes en un curso universitario. En L. Orsi (coord.) (2019). VI Jornadas de Investigación en Humanidades: homenaje a Cecilia Borel. Bahía Blanca, Argentina. 383-387

Coulthard, M., \& Brazil, D. (1981). Exchange structure. En M. Coulthard y M. Montgomery (Ed.), Studies in discourse analysis (pp.82-106). London: Routledge.

Gutiérrez-Giraldo, M. C., \& López-Isaza, G. A. (2011). La interactividad en la construcción del conocimiento escolar. Innovar, 21(41), 5-18.

Hall, J. K., \& Walsh, M. (2002). Teacher-student interaction and language learning. Annual Review of Applied Linguistics, 22, 186.

Jacknick, C. M. (2011). "But this is writing": Post-Expansion in Student-Initiated Sequence. Novitas-Royal (Research on youth and language), 5(1), 39-54.

Jara-Gutiérrez, N. P., Díaz-López, M. M., \& Zapata-Castañeda, P. N. (2015). Desafíos educativos para el profesor de medicina: Evaluación de su desempeño. latreia, 28(3), 292-299.

Lazcano, X., Valenzuela, J. S. C., \& Conget, P. (2013). Teorías implícitas sobre la enseñanza y su asociación con las prácticas pedagógicas de los docentes de la carrera de Medicina. Revista de Educación en Ciencias de la Salud, 10(1), 47-52.

Lemke, J. L. (1990). Talking Science. Language, learning, and Values. Norwood, NJ: Ablex.

Li, H. (2013). Student Initiatives and Missed Learning Opportunities in an IRF Sequence: A Single Case Analysis. L2 Journal, 5(1), 68-92.

Lin, A. M. (2007). What's the use of "triadic dialogue"?: Activity theory, conversation analysis, and analysis of pedagogical practices. Pedagogies: An International Journal, 2(2), 77-94.

Mehan, H. (1979). Learning lessons. Social organization in the classroom. Cambridge, Mass.: Harvard University Press.

Nassaji, H., \&Wells, G. (2000). What's the use of "triadic dialogue"?: An investigation of teacher-student interaction. Applied Linguistics, 21(3), 376-406.

Nunn, R. (2001). Language learning across boundaries. Negotiating classroom rituals. Teaching English as a second foreign language, 5(2). Recuperado de http://www. tesl-ej.org/wordpress/issues/volume5/ej18/ej18a1/?wscr.

Park, J. E. (2020). Exploring the Assumptions that Underlie Knowledge Elicitation in Classroom Interaction. Journal of Research, 24(1), 127-136.

Pozo, J. I. (2014). Psicología del aprendizaje humano: Adquisición de conocimiento y cambio personal. Madrid: Morata.

Quera, V. (1993). Análisis secuencial. En M. T. Anguera (Ed.). Metodología observacional en la investigación psicológica, vol. II. Barcelona: PPU.

Ruiz, E., González-Celis, A., Cruz, J., \& Hernández, D. (2018). Validación de contenido de un cuestionario profesor-alumno. Enseñanza e Investigación en Psicología, 23(3), 260-270.

Sackett, G. P. (1980). An initial investigation of the usability of conversational data for doing Sociology. En D. Sudnow (ed.), Studies in Social Interactions. New York: Free Press.

Sackett, G. P. (1987). Analysis of sequential social interaction data: Some issues, recent developments, and causal inference model. En: J. Osofsky (ed.), Handbook of infant development. (pp. 855-878). New York: Wiley.

Seedhouse, P. (2004). The Interactional Architecture of the Language Classroom: A Conversation Analysis Perspective. Malden, MA: Blackwell.

Sert, O., \& Seedhouse, P. (2011). Introduction: Conversation Analysis In Applied Linguistics. Novitas-Royal (Research on Youth and Language), 5 (1), 1-14.

Sinclair, J., Mc H., \& Coulthard, R. M. (1975). Towards an Analysis of Discourse. The English used by teachers and pupils. Oxford: Oxford University Press.

Sociedad Mexicana de Psicología (2010). Código Ético. México: Trillas. Stierer, B., \& Maybin, J. (eds.) (1994). Language, Literacy and Learning in Educational Practice. Clevedon: Multilingual Matters.

Tharp, R., \& Gallimore, R. (1989). Rousing Minds to Life: Teaching, Learning, and Schooling in Social Context. American Educator.

Tronchoni, A (2019). Estudio observacional de la comunicación multimodal en el aula universitaria: Contextos y estructuras de participación discursiva en las sesiones magistrales (tesis doctoral). Barcelona: Universitat de Barcelona. Facultat de Psicologia.

Urrutia-Aguilar, M. E., \& Guevara-Guzmán, R. (2013). Estrategias docentes en el primer año de la carrera de Médico Cirujano y nivel de aprovechamiento académico. Investigación en educación médica, 2(6), 77-81.

Villalta-Páucar, M. A., \& Martinic-Valencia, S. (2013). Interacción didáctica y procesos cognitivos. Una aproximación desde la práctica y discurso del docente. Universitas Psychologica, 12(1), 221-233.

Villalta-Páucar, M. A., Martinic-Valencia, S., \& Guzmán.Droguett, M. A. (2011). Elementos de la interacción didáctica en la sala de clase que contribuyen al aprendizaje en contexto social vulnerable. Revista Mexicana de Investigación Educativa, 16(51), 1137-1158.

Waring, H. Z. (2009). Moving out of IRF (Initiation-Response-Feedback): A single-case analysis. Language Learning, 59 (4), 796-824.

Waring, H. Z. (2011). Learner initiatives and learning opportunities in the language classroom. Classroom Discourse, 2(2), 201-218.

Waring, H. Z., Reddington, E., \& Tadic, N. (2016). Responding artfully to student-initiated departures in the adult ESL classroom. Linguistics and Education, 33, 28-39.

Wells, G. (1999). Dialogic Inquiry: Towards a sociocultural practice and theory of education. Cambridge: Cambridge University Press.

Wells, G., \& Mejía-Arauz, R. (2005). Hacia el diálogo en el salón de clases: Enseñanza y aprendizaje por medio de la indagación. Sinéctica, Revista Electrónica de Educación (26), 1-19. 


\section{Anexos}

\section{Anexo 1.}

Muestra del inventario de interacción profesor-alumno (IIP-A).

\section{Inventario de Interacción Profesor-Alumno}

Grupo: Semestre: Edad: Género:

Instrucciones: A continuación se presenta una lista de 31 afirmaciones de las que deberás seleccionar la opción que mejor refleje tu opinión sobre la actividad en el salón de clases. Coloca una X según sea el caso en la casilla correspondiente empleando la siguiente escala:

4 - Totalmente de acuerdo

3 - De acuerdo

2 - En desacuerdo

1 - Totalmente en desacuerdo

\section{No.} Í́TEMS

1 El profesor imparte los temas de la asignatura en un orden que facilita su seguimiento.

2 El profesor mantiene un ritmo de exposición adecuado.

3 El profesor demuestra interés por la materia que imparte.

4 El profesor clarifica la importancia de los contenidos del curso.

5 El profesor distribuye el tiempo entre los temas según su dificultad.

6 El profesor relaciona los nuevos conceptos con otros conocidos.

7 El profesor contrasta varias teorías relacionadas con la asignatura.

8 El profesor relaciona los conceptos teóricos con ejemplos, ejercicios y problemas.

9 Las explicaciones del profesor me han ayudado a entender mejor la asignatura.

10 El profesor procura que los estudiantes apliquen los conceptos adquiridos en la asignatura.

11 El profesor genera interés por la asignatura a los alumnos

12 El profesor motiva a sus alumnos a cursar materias con el

13 El profesor fomenta la participación de los alumnos.

14 El profesor consigue que los estudiantes participen activamente en sus clases.

15 El profesor brinda oportunidades para participar activamente en sus clases.

16 El profesor introduce temas de discusión y anima a los estudiantes a participar.

17 El profesor hace preguntas interesantes y estimulantes en clase.

18 El profesor resuelve las dudas con exactitud.

19 El profesor procura saber si los alumnos entienden lo que explica.

20 El profesor busca la forma de que los estudiantes respondan sus preguntas.

21 El profesor adapta sus estrategias para afrontar situaciones imprevistas.

22 El profesor se muestra dispuesto a ayudar a los estudiantes que tienen dificultades.

23 El profesor proporciona la posibilidad de conocer y comentar la valoración de las evaluaciones.

24 El profesor motiva a sus alumnos a trabajar al máximo.

25 El profesor hace un seguimiento del aprendizaje a lo largo del curso. 
Meta-Análisis del Artículo 


\section{Dimensión Cuantitativa}

\section{Perfil de Evaluación entre pares}
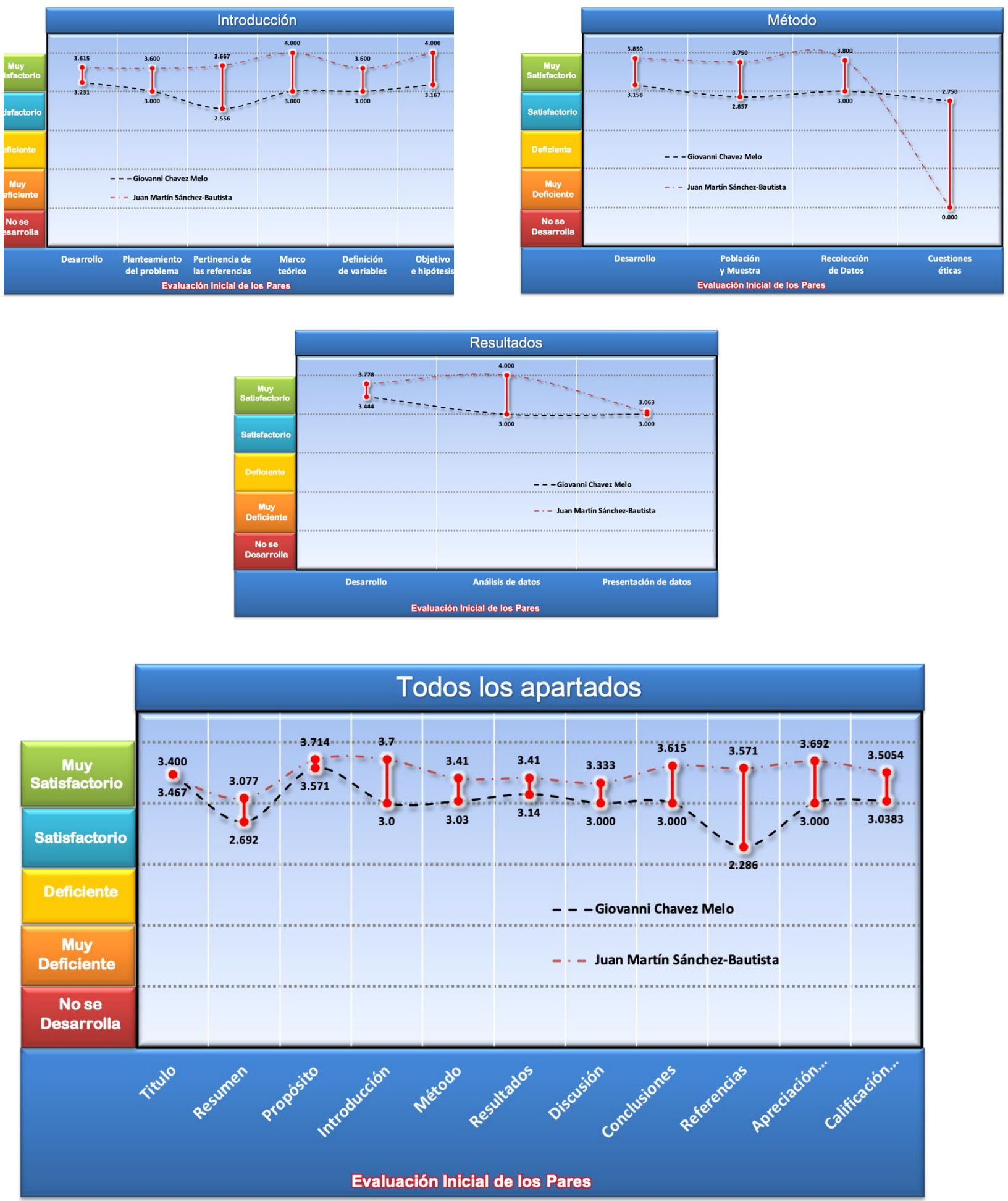


\section{Índice de Concordancia}

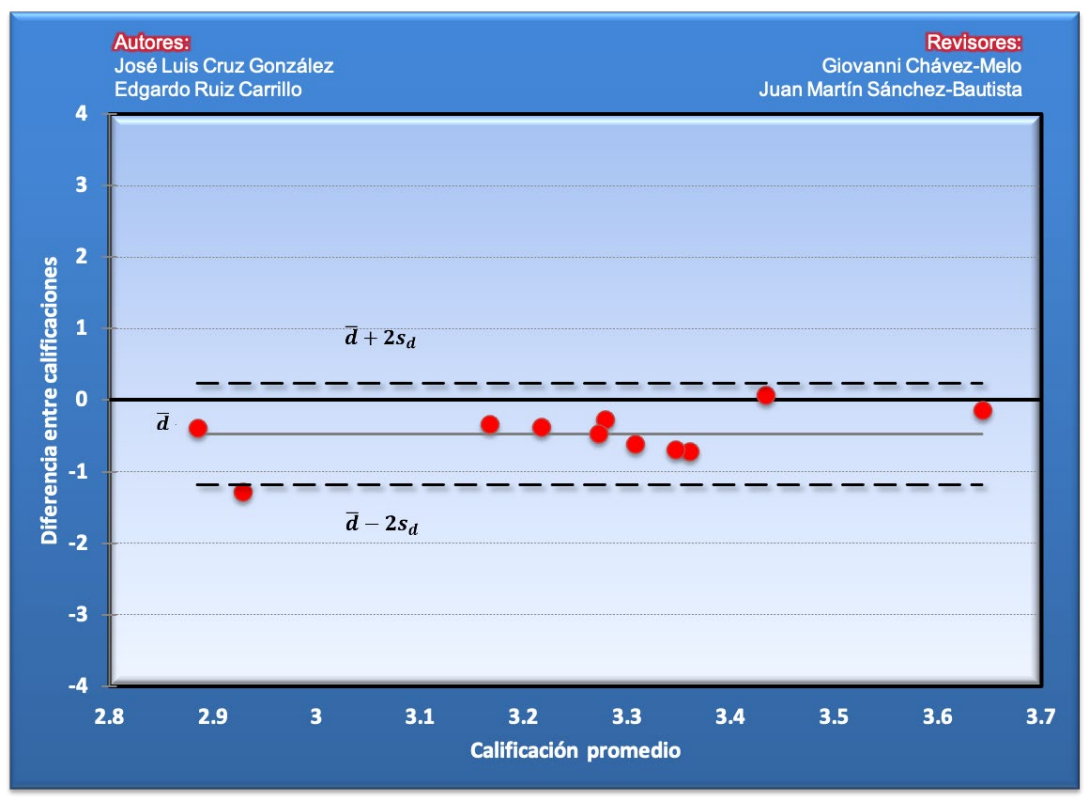

Índice de Acuerdo

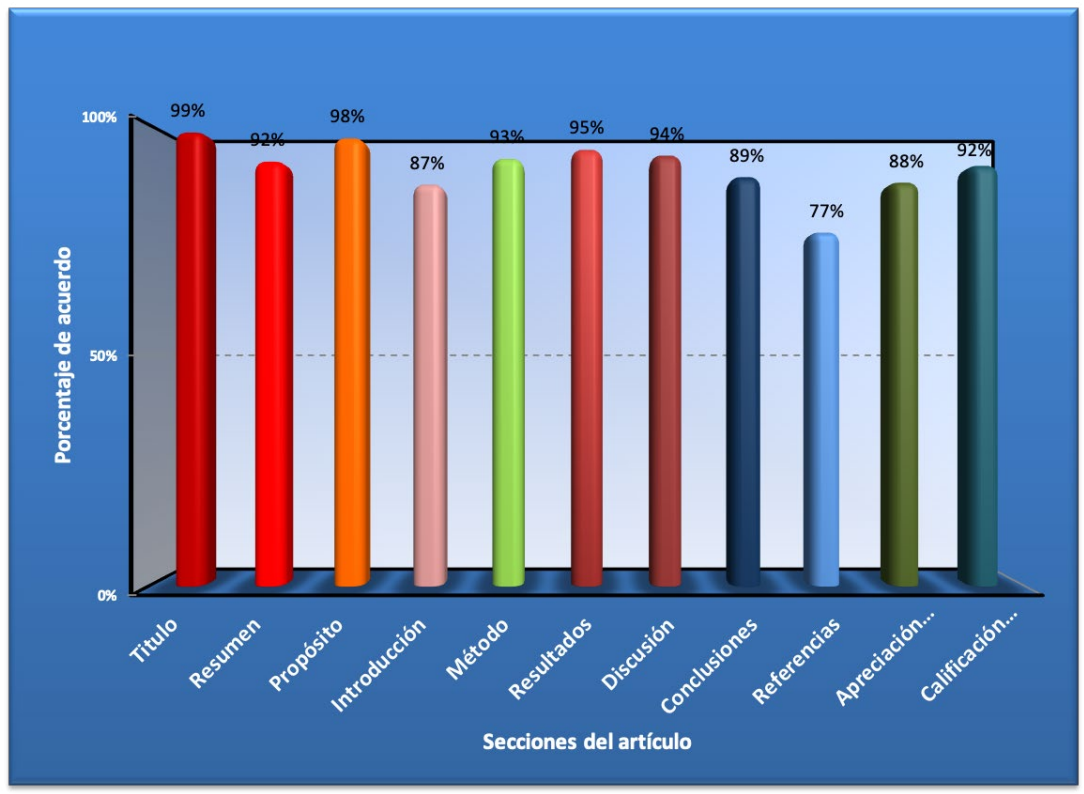




\begin{tabular}{|c|c|}
\hline Revisor 1 & Revisor 2 \\
\hline Giovanni Chavez-Melo & Juan Martín Sánchez-Bautista \\
\hline \multicolumn{2}{|c|}{ Título/Autoría } \\
\hline $\begin{array}{l}\text { El término "clases" puede tener un significado diferente } \\
\text { en disciplinas de ciencias sociales, puede referirse a clases } \\
\text { sociales, se sugiere cambiar por algún sinónimo. }\end{array}$ & $\begin{array}{l}\text { Se sugiere las siguientes forma para ajustarse al requisitc } \\
\text { de } 15 \text { palabras "Estrategias discursivas y opinión de alum } \\
\text { nos de Medicina sobre la interacción con el docente" "Es } \\
\text { trategias discursivas y opinión de alumnos que estudiar } \\
\text { Medicina sobre la interacción con el docente" Solo incluye } \\
\text { el correo de uno de los autores }\end{array}$ \\
\hline
\end{tabular}

\section{Resumen}

Se sugiere la siguiente redacción "Los participantes fueron alumnos de cinco grupos de la carrera de medicina de una institución Educativa de Educación Superior (IPES)

Se requiere reducir la longitud del resumen a 150 palabras e integrar al menos una palabra clave. Revisar la redacción del resumen en inglés, la corrección de algunas palabras mejorarán el sentido de la redacción.

y cinco profesores que impartían asignaturas teóricas en sustitución de la original "La investigación contó con la participación de cinco grupos consistentes de cinco profesores de asignaturas teóricas de la carrera de Medicina de una Institución Educativa de Educación Superior (IPES), y los alumnos correspondientes a sus grupos" por considerar que así se mejor ala redacción.

\section{Próposito del Estudio}

Se sugiere proporcionar mayores detalles del método Se sugiere que defina el uso de los conceptos de perceputilizado. ción y opinión para homogeneizar su redacción.

\section{Introducción}

La revisión de literatura a la que hace mención no se encuentra actualizada, la gran mayoría de las fuentes son de 5 años o más de antigüedad, no se abarcan las investigaciones recientes relacionadas con el tema de investigación Algunos planteamientos de la introducción son presentados de una manera que serían más adecuados en la discusión en la disciplina que aborda.

\section{Método}

Los totales de la Tabla 1: Estadística de los participantes por grupo que corresponde a hombres y mujeres no es consistente. Se sugiere la integración del cuestionario IIP-A para la replicabilidad del estudio.

Desarrollar los mecanismos de validación de las categorías construidas No se explica como se seleccionaron las sesiones a observar

\section{Resultados}

Ya se había comentado que los datos de la Tabla 1 no presentan consistencia en los totales.

Los códigos en la tabla hacen poco accesible leer los datos. 
Artículo Teórico | Estrategias discursivas y opinión del alumnado... Cruz-González ơ Ruíz Carrillo

\begin{tabular}{|l|l|}
\hline \multicolumn{1}{|c|}{ Revisor 1 } & \multicolumn{1}{|c|}{ Revisor 2 } \\
\hline \multicolumn{1}{|c|}{ Discusión } \\
\hline $\begin{array}{l}\text { La discusión que se aborda es crítica y profunda res- } \\
\text { pecto a los resultados y la teoría. }\end{array}$ & $\begin{array}{l}\text { Se sugiere considerar discutir sobre porcentajes más que } \\
\text { sobre frecuencias }\end{array}$ \\
\hline \multicolumn{2}{|c|}{ Conclusiones } \\
\hline $\begin{array}{l}\text { Se sugiere abordar con mayor detalle la aportación de los } \\
\text { hallazgos. }\end{array}$ & - \\
\hline \multicolumn{2}{|c|}{ Referencias } \\
\hline $\begin{array}{l}\text { Falta consistencia en las citas, algunas aparece "\&" y en } \\
\text { otras “y" cuando se menciona a más de un autor. Existe } \\
\text { un gran número de citas y referencias que no se encuen- } \\
\text { tran disponibles en el documento o en el listado de re- } \\
\text { ferencias. }\end{array}$ & buscar referencias actualizadas \\
\hline
\end{tabular}


